\title{
Peertechz
}

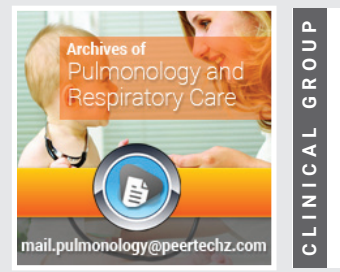

\section{Respiratory Management of Myotonic Dystrophy}

\author{
John R Bach ${ }^{1 *}$, Giulio Chiarello ${ }^{2}$ and Somnath Gonapa ${ }^{3}$ \\ ${ }^{1} \mathrm{MD}$, Professor of Physical Medicine and Rehabilitation, Professor of Neurology, Department of \\ Physical Medicine and Rehabilitation, Rutgers University, New Jersey Medical School, Medical Director \\ of the Center for Ventilator Management Alternatives and Pulmonary Rehabilitation of the University \\ Hospital of New Yark, New Jersey, USA \\ ${ }^{2}$ Registered Respiratory Therapist, Department of Physical Medicine and Rehabilitation, Rutgers \\ University-New Jersey Medical School, New Yark, New Jersey, USA \\ ${ }^{3}$ MS4 Rutgers University, New Jersey Medical School, USA
}

Received: 16 April, 2020

Accepted: 05 May, 2020

Published: 06 May, 2020

*Corresponding author: John R Bach, MD, Professor of Physical Medicine and Rehabilitation, Professor of Neurology, Department of Physical Medicine and Rehabilitation, Rutgers University, New Jersey Medical School, Medical Director of the Center for Ventilator Management Alternatives and Pulmonary Rehabilitation of the University Hospital of New Yark, New Jersey, USA, E-mail: bachjr@njms.rutgers.edu

https://www.peertechz.com

\section{Check for updates}

\section{Abstract}

Patients with myotonic dystrophy can avoid episodes of respiratory failure by using up to continuous noninvasive ventilatory support (CNVS). Of 154 patients consecutively presenting to a neuromuscular disease clinic, 143 had types 1 or 2 and 11 had congenital myotonic dystrophy. Of the 76 who came multiple times, 41 were prescribed and used at least sleep NVS to relieve symptoms of fatigue and hypersomnolence. They had sleep and end-tidal (Pet) CO2 with highs over $50 \mathrm{~mm} \mathrm{Hg}$ and many $4 \%$ or greater 02 desats per hour of sleep and all had mean sleep 02 sat levels less than $95 \%$ before beginning sleep NVS. Thirteen eventually had to extend NVS use into and eventually throughout daytime hours for CNVS. While using NVS during the day their arterial blood gases were within normal limits. When the 41 were using NVS only during sleep, 24 of the 41 also had normalization of diurnal SpO2 to $\geq 95 \%$ and decreased hypercapnia. The 41 patients have been using sleep NVS for $6.70 \pm 3.64$ (range $=0.75$ to 19 ) years per patient or for 274.7 patient-years. Seventy-two have died or been lost to follow-up. The 13 up to CNVS users have been doing so for 75.7 patient-years or a mean of $5.8 \pm 3.2$ (range $=0.2$ to 10 ) years per patient. The VC from first visit to most recent visit of all 71 decreased a mean $49.3 \pm 168.8 \mathrm{ml}$ or about $1.5 \%$ per year. it decreased a mean $52 \pm 171$ per year for the $41 \mathrm{NVS}$ users over $274.7 \pm 3.64$ patient-years.

\section{Abbreviations}

ABG: Arterial Blood Gases; CNS: Central Nervous System; CNVS: Continuous Noninvasive Ventilatory Support; CPF: Cough Peak Flows; DMD: Duchenne Muscular Dystrophy; EPAP: Expiratory Positive Airway Pressure; IPAP: Inspiratory Positive Airway Pressure; VC: Vital Capacity; LVR: Lung Volume Recruitment; MIC: Maximum Insufflation Capacity; NVS: Noninvasive Ventilatory Support; PetCO2: End-Tidal Carbon Dioxide; Sp02: Pulse Oxyhemoglobin Saturation; VFBA: Ventilator Free Breathing Ability

\section{Introduction}

Myotonic dystrophy has many unique aspects. Virtually all patients complain of hypersomnolence and fatigue. While both can be at least in part relieved by Noninvasive Ventilatory Support (NVS), some hypersomnolence usually remains and may be due to Central Nervous System (CNS) pathology. The chemosensitivity of the respiratory centers (the Po.1) is well preserved in patients with myotonic dystrophy, as it is for patients with other muscular dystrophies. However, even in the absence of weak respiratory muscles, output to breathing is modulated by impaired respiratory mechanics caused by myotonia. The myotonia causes chronic symptomatic alveolar hypoventilation despite Vital Capacities (VCs) and Maximum Insufflation Capacities (MICs) that may be well preserved for many years [1]. Even in the absence of significant central or obstructive sleep apneas [2,3], or chronic diurnal hypercapnia, these patients can exhibit sleep hypercapnia and hypersomnia. Apneas and hypopneas, when present, can be exacerbated by chronic alveolar hypoventilation due to weak and myotonic inspiratory muscles. Even if the respiratory muscles are relatively strong, the myotonia makes it difficult for them to relax and reverse direction, decreasing lung ventilation and leading to hypercapnia.

Despite severe hypercapnia during sleep, because these patients are usually able to walk, because of their frequent general apathy and cognitive impairment, and because of the myotonic nature of the muscle impairment by which they may not feel great benefit by using Noninvasive Ventilatory 
Support (NVS), they very often fail to use NVS sufficiently either during daytime hours or overnight to maintain normal Arterial Blood Gases (ABGs). One study reported poor compliance with ventilator use by $62 \%$ of 38 patients by comparison with patients with other NMDs [4]. Thus, with many patients not benefiting optimally from NVS, they can be hospitalized repeatedly before succumbing from cor pulmonale and cardiorespiratory failure. Nevertheless, we suspect that those who do cooperate to use at least nocturnal NVS may have improved ABGs, symptoms, and survival [5]. It may be that the weaker patients, those wheelchair dependent with very low VCs, have better prognoses than ambulatory patients who do not appreciate the benefits of NVS and use it sparingly. Ambulatory hypercapnic myotonic dystrophy patients neither want to carry nor pull a ventilator with them while they walk nor do they feel so much benefit as those in wheelchairs who literally can not breathe without up to continuous NVS $[6,7]$.

The objective of this retrospective longitudinal study is to determine changes in VC in populations of patients with myotonic dystrophy that use NVS and active lung volume recruitment vs. those who do not.

\section{Methods}

This is a retrospective longitudinal study of consecutively presenting patients to a neuromuscular disease clinic. Besides demographic information, vital capacity measured in sitting and supine positions, lowest observed value of $\mathrm{SpO}$, highest observed PetCO2, maximum insufflation capacity, and Cough Peak Flows (CPF) were recorded for every visit. Patients using low span bi-level PAP were switched to NVS settings on bilevel machines, e.g. inspiratory PAP (IPAP) $20 \mathrm{cmH} 2 \mathrm{O}$, EPAP 4 $\mathrm{cmH} 2 \mathrm{O}$, or to portable ventilators volume preset from 700 to $1500 \mathrm{ml}$. Hours per day of use of NVS was noted, as was if the patient underwent tracheotomy.

\section{Results}

Of 154 patients consecutively presenting to a neuromuscular disease clinic with DNA confirmed myotonic dystrophy, 143 had types 1 or 2 and 11 congenital myotonic dystrophy. Of the 143, 67 came only once. Of the 11 congenital patients, 9 came only once and the other two were not placed on or did not use NVS. Of the 76 who came multiple times, 41 were prescribed and used at least sleep NVS on the basis of symptoms of hypersomnolence, fatigue, and morning headaches. They also had sleep end-tidal (Pet) $\mathrm{CO} 2$ averaging 47 or more $\mathrm{mmHg}$ with highs over 50 $\mathrm{cmH} 2 \mathrm{O}$ and many $4 \%$ or greater $\mathrm{O}_{2}$ desats per hour of sleep. All had mean 02 sat levels less than $95 \%$. Thirteen of the 41 , when feeling dyspneic upon discontinuing NVS in the morning, extended NVS use into and eventually throughout daytime hours. Several lost all ventilator free breathing ability (VFBA). While using NVS during the day their ABGs were within normal limits. When the 41 were using NVS only during sleep, 24 of the 41 also had normalization of diurnal oxyhemoglobin saturation (SpO2) to $\geq 95 \%$ and decreased hypercapnia. The others had normal diurnal $\mathrm{SpO} 2$ levels before beginning sleep NVS but their diurnal PetCO2 decreased from $46 \pm 1.8$ to $42 \pm 1.9 \mathrm{mmHg}$. Wash out of the end-tidal samples due to the nasal NVS made nocturnal PetCO2 quantitations unreliable. Nevertheless, all 41 patients reported symptomatic improvements. The 41 patients have been using and continue to use NVS for $274.7 \pm 3.64$ patientyears, at least $6.70 \pm 3.64$ (range $=0.75$ to 19 ) years per patient and seventy-two have died or been lost to follow-up. The 13 up to CNVS users have been doing so for 75.68 patient-years or a mean of $5.82 \pm 3.27$ (range $=0.2$ to 10 ) years per patient. The VC from first visit to the most recent visit of all 71 managed by NVS decreased a mean $49.3 \pm 168.8 \mathrm{ml}$ or about $1.5 \%$ per year whereas normal decrease is $1 \%$ for males, $1.2 \%$ for females or about $32 \mathrm{ml}$ per year after age 20 . It decreased a mean $52 \pm 171$ for the 41 NVS users over $274.7 \pm 3.64$ patient-years.

For 17 patients beginning NVS at the first visit to the second visit a mean $7 \pm 6.9$ (range $=1$ to 29 ) months later the VC increased by $165.2 \pm 99.5$ (range $=20$ to 330 ) $\mathrm{ml}$. However, it also increased by $176 \pm 107.14$ (range $=70$ to 340 ) $\mathrm{ml}$ for 5 of the 30 who did not use sleep NVS but who performed two to three times daily active lung volume recruitment (LVR) from the first visit to the second visit $18.2 \pm 13.16$ months later. At least 7 NVS users reported relief of echocardiographic evidence of right ventricular strain. Five of the non-NVS users underwent tracheotomies in their local hospitals during episodes of cardiopulmonary failure (Table 1 ).

\section{Discussion}

The fact that nine myotonic dystrophy patients underwent tracheotomies locally is in contrast to only 2 of our 279 NVS users with Duchenne Muscular Dystrophy (DMD), none of whom having failed extubation attempts but who underwent emergency tracheotomies during failed intubation attempts. This suggests that the myotonic dystrophy patients did not use NVS adequately to avoid ARF and resort to tracheotomies even though their VCs were much greater than those of the DMD patients, over 100 of whom had VCs less than $250 \mathrm{ml}$.

Although there are no long term studies on the use of mechanical ventilation by myotonic dystrophy patients, we have managed a handful using Continuous Tracheostomy Mechanical Ventilation (CTMV), and 8 others using CNVS with no VFBA for a prolongation of survival of at least 8 years. In a recent study of 13 hypercapnic patients with some combination of hypersomnolence, dyspnea, morning headaches, and sleep disturbance using nocturnal nasal or oronasal "NIV" which included Continuous Positive Airway Pressure (CPAP) and less than NVS support settings of bi-level PAP, diurnal $\mathrm{PaCO} 2$ decreased from 64 to $53 \mathrm{mmHg}, \mathrm{PaO} 2$ increased from 53 to 65 $\mathrm{mmHg}$, mean nocturnal $\mathrm{O2}$ sat increased from 80.5 to $90.3 \%$, and transcutaneous PCO2 decreased from 59.3 to $41.4 \mathrm{mmHg}$. Three ventilator users died but the other 10 used bi-level PAP for at least 27 months [5].

In another study 32 adult patients diagnosed with sleepdisordered breathing began bi-level PAP with inspiratory PAPs of 8.5 to $18.7 \mathrm{~cm} \mathrm{H2O}$, expiratory PAP not noted. At 2 year follow-up diurnal $\mathrm{PaCO} 2$ was stable at $41.5 \mathrm{mmHg}$; $\mathrm{PaO} 2$ decreased from 79.2 to $76.6 \mathrm{~mm} \mathrm{Hg}$, mean nocturnal 02 sat increased from 91.7 to $93.8 \%$, and mean transcutaneous $\mathrm{PCO} 2$ decreased from 46.4 to $40.1 \mathrm{mmHg}$ [8]. 
Table 1: Extent of use of noninvasive ventilatory support.

\begin{tabular}{|c|c|c|c|c|c|c|c|}
\hline $\begin{array}{c}\text { Myotonic Distrophy } \\
\text { Patients }\end{array}$ & Males & Females & Mean age \pm s.d. & $\begin{array}{c}\text { Mean Vital Capacity }(\mathbf{m l}) \\
\text { sitting first visit } \pm \text { s.d. }\end{array}$ & $\begin{array}{c}\text { Years from first visit to } \\
\text { last visit } \\
\text { (mean } \pm \text { s.d.) }\end{array}$ & $\begin{array}{c}\text { Mean Vital Capacity } \\
\text { (ml) sitting } \\
\text { most recent } \pm \text { s.d. }\end{array}$ & $\begin{array}{c}\text { Cough Peak flows } \\
\text { L/min } \\
\text { (mean } \pm \text { s.d.) }\end{array}$ \\
\hline Didn't Use NVS & 23 & 7 & $32.6 \pm 14.1$ & $3311.3 \pm 1146.1$ & $5.5 \pm 5.2$ & $3213.6 \pm 1044.2$ & $5.6 \pm 1.2$ \\
\hline Sleep-only NVS & 15 & 13 & $40.4 \pm 11.8$ & $2618 \pm 718.3$ & $5.1 \pm 3.8$ & $1780 \pm 703.3$ & $5.1 \pm 1.4$ \\
\hline Sleep plus daytime NVS & 8 & 5 & $45.1 \pm 15.3$ & $1610.7 \pm 679.7$ & $3.8 \pm 3.3$ & $1397.7 \pm 627.2$ & $4 \pm 1.4$ \\
\hline
\end{tabular}

Another study considered 12 patients using bi-level PAP. Investigating the effects of a 1-month withdrawal followed by a 1-month reinstitution of bi-level PAP, settings not reported, initial diurnal PaCO2 $43.1 \mathrm{~mm} \mathrm{Hg}$ increased to $46.3 \mathrm{~mm} \mathrm{Hg}$ after the one month withdrawal then decreased back to 43.9 $\mathrm{mm} \mathrm{Hg}$ after one month of reinstitution; $\mathrm{PaO} 283.6 \mathrm{~mm} \mathrm{Hg}$ at baseline decreased to $76.9 \mathrm{~mm} \mathrm{Hg}$ after one month withdrawal then increased to $80.9 \mathrm{~mm} \mathrm{Hg}$ after one month of reinstitution. Mean nocturnal $\mathrm{O}_{2}$ sat $95.0 \%$ at baseline decreased to $92.2 \%$ after one month withdrawal then increased to $95.1 \%$ after one month of reinstitution [9]. None of these studies described patients who, with time, became dependent on continuous ventilatory support. All our 13 patients using CNVS had normal $\mathrm{CO} 2$ and $\mathrm{O} 2$ sat levels while using it and the levels deteriorated and respiratory distress developed upon discontinuation of CNVS, including immediately for 8 patients.

A study of mortality in myotonic dystrophy over a 47 year period indicated a median survival of 60 years for males and 59 years for females with survival to age 65 only $18 \%$ by contrast to the normal $78 \%$. The causes of death were about $30 \%$ from pneumonia and $30 \%$ from cardiac arrhythmias. Half of the patients were wheelchair-bound [10]. Inadequate ventilatory and cough assistance most probably played a big role in the deaths due to pneumonia, cor pulmonale, heart failure and fatal arrthythmias. These patients have periods when their CPF are very poor and they need access to MIE to avoid pneumonia.

Using NVS settings to normalize $\mathrm{CO} 2$ and $\mathrm{O} 2$ sat levels as well as AHI, rather than only low pressure support bi-level PAP, better improves blood gases and can prolong life by CNVS when needed. Some patients are being told that by polysomnography they have no evidence of sleep disordered breathing and do not require treatment despite severe hypercapnia that was not monitored but that we observed on presentation to us. Apnea-hypopnea indices are often normal or normalized by noninvasive ventilation at less than NVS settings despite severe sleep hypercapnia that can result in cor pulmonale.

On conclusion, it is clear that occasional patients with myotonic dystrophy can become CNVS dependent, as an alternative to undergoing tracheotomy, and this can occur without hospitalization or an episode of acute respiratory failure.

\section{References}

1. Begin R, Bureau MA, Lupien L, Lemieux B (1980) Control and modulation of respiration in Steinert's myotonic dystrophy. Am Rev Respir Dis 121: 281-289. Link: https://bit.ly/35sdFHo

2. Manni R, Zucca MR, Martinetti C, Martinetti M, Ottolini A, Lanzi G, et al (1991) Hypersomnia in dystrophia myotonica: a neurophysiological and immunogenetic study. Acta Neurol Scand 84: 498-502. Link: https://bit.ly/35sEnQ8

3. Lopez Esteban P, Peraita Adrados R (2000) Sleep and respiratory disorders in myotonic dystrophy of Steinert. Neurologia 15: 102-108. Link: https://bit.ly/3fkylA6

4. Tejero G, Langevin B, Petitjean T, et al. (2001) Long term home mechanica ventilation in myotonic dystrophy: follow up and compliance. $8^{\text {th }}$ Journées Internationales de Ventilation á Domicile, abstract 129, March 7, 2001, Hôpital de la Croix Rousse, Lyon, France.

5. Nugent AM, Smith IE, Shneerson JM (2002) Domiciliary assisted ventilation in patients with myotonic dystrophy. Chest 121: 459-464. Link: https://bit.ly/35AAvN9

6. Pinto TC, Winck JC, Gonçalves MR (2019) Ventilatory support via mouthpiece to facilitate ambulation. Am J Phys Med Rehabil 98: 789-793. Link: https://bit.ly/3fkzlcW

7. de Die-Smulders CE, Höweler CJ, Thijs C, Mirandolle JF, Anten HB, et al. (1998) Age and causes of death in adult-onset myotonic dystrophy. Brain 121: 15571563. Link: https://bit.ly/2YDI1oS

8. Spiesshoefer J, Runte M, Heidbreder A, Dreher M, Young P, et al. (2019) Sleepdisordered breathing and effects of non-invasive ventilation on objective sleep and nocturnal respiration in patients with myotonic dystrophy type I. Neuromuscul Disord 29: 302-309. Link: https://bit.ly/2Ws4ltt

9. O’Donoghue FJ, Borel JC, Dauvilliers Y, Levy P, Tamisier R, et al. (2017) Effects of 1-month withdrawal of ventilatory support in hypercapnic myotonic dystrophy type 1. Respirology 22: 1416-1422. Link: https://bit.ly/2WORDbF

10. Hill NS, Eveloff SE, Carlisle CC, Goff SG (1992) Efficacy of nocturnal nasal ventilation in patients with restrictive thoracic disease. Am Rev Respir Dis 145: 365-371. Link: https://bit.ly/2Ws4PoT 